\title{
Enfoques analíticos y programas educativos para gestionar la convivencia escolar en México
}

\section{Analytical approaches and educational programs to manage school coexistence in México}

\author{
María Concepción Chávez Romo ${ }^{1}$ \\ Universidad Pedagógica Nacional, México
}

\begin{abstract}
Resumen. Este artículo tiene como objetivo recapitular tres modos de acercamiento a la comprensión de lo que sucede en la vida de las escuelas de educación básica en México en relación con el desafío de aprender a vivir juntos: convivencia, disciplina y violencia escolar. Se trata de distintas orientaciones temáticas identificadas en las investigaciones educativas desde los años 1990 que podrían consolidarse como perspectivas analíticas complementarias para comprender la complejidad de los problemas de la convivencia escolar, siempre y cuando su desarrollo sea equilibrado y sistemático. Sin embargo, esto no parece estar sucediendo, al menos en el caso de las investigaciones realizadas en la primera década del siglo XX (entre los años 2002 y 2011) se observa un mayor interés por analizar el fenómeno de la violencia entre estudiantes. Paralelamente, a esta diversidad de acercamientos existen también programas educativos derivados de la actual política educativa que destacan la promoción de ambientes escolares seguros y libres de violencia, pero la mayor parte de las acciones están dirigidas a la prevención y a la reducción de la violencia entre estudiantado, lo cual es relevante destacarlo para valorar los alcances y las limitaciones en la comprensión y transformación de prácticas de convivencia y de resolución de conflictos en las escuelas.
\end{abstract}

Palabras clave. Convivencia escolar, disciplina escolar, programa nacional de convivencia escolar, escuela segura, cultura de la legalidad

\begin{abstract}
This article aims to recapitulate three modes of approach to understanding what happens in the life of schools in relation to the challenge of learning to live together: coexistence, discipline and school violence. These are different thematic orientations identified in educational research since the 1990s that could be consolidated as complementary analytical perspectives to understand the complexity of life in schools, especially if a balanced and systematic development is achieved. However, this does not seem to be happening. In the research conducted in the first decade of the twentieth century (between 2002 and 2011) there is a greater interest in analyzing the phenomenon of violence among students. Along with this diversity of approaches there are also educational programs derived from the current educational policy that emphasize the promotion of safe and violence-free school environments, but most of the actions are aimed at the prevention and reduction of violence among students, which is relevant to assess the scope and limitations in the understanding and transformation of coexistence practices and conflict resolution in schools.
\end{abstract}

Keywords. Coexistence and school discipline, national program of school coexistence, safe school, culture of legality

\footnotetext{
${ }^{1}$ Doctora en Pedagogía, especialista en procesos de formación docente, elaboración de materiales educativos en los temas de educación para la paz y los derechos humanos, formación en valores, cultura de la legalidad y formación ciudadana. Dirección electrónica: $\underline{\text { cchavezr@g.upn.mx }}$
} 


\section{Introducción}

Las reflexiones que se exponen en los apartados siguientes están orientadas por dos preguntas centrales: ¿cuáles son las vertientes analíticas que favorecen la exploración y la comprensión de lo que acontece en las escuelas en materia de convivencia? y ¿qué programas $\mathrm{o}$ acciones educativas se han desarrollado en el marco estatal como respuesta a los desafíos de la convivencia, disciplina y violencia escolar?

En ese sentido, el presente aporte resulta de una revisión documental que pretende mostrar las principales tendencias identificadas en la producción de investigaciones educativas mexicanas, las cuales reúnen temas interconectados como convivencia, resolución de conflictos, disciplina, indisciplina, seguridad y violencia escolar. Los tres enfoques analíticos que sirven de base para comprender estos asuntos en la vida de las escuelas de educación básica son los siguientes: convivencia, disciplina y violencia escolar; sobre ellos se abundará en la primera parte del artículo.

Se acotan las reflexiones al ámbito de la educación básica que incluye los niveles de educación preescolar (tres años), primaria (seis años) y secundaria (tres años) porque a este pertenece la mayoría de las investigaciones efectuadas. Se destaca que, en la mayoría de las investigaciones educativas mexicanas, así como en algunos programas educativos nacionales, generados aproximadamente en la primera década del siglo XXI, se le ha dado prioridad a la comprensión de la violencia escolar vinculada con conflictos y divergencias entre estudiantes (bullying y acoso escolar). Esto ha limitado la sistematización, la comprensión y la interpretación integral del sentido de las interacciones y la experiencia de los involucrados en el proceso educativo. Para ello, se toma como referencia básica los dos estados del conocimiento que se han producido en México durante los periodos 1992-2002 (Furlán, 2003) y 2002-2011 (Furlán y Spitzer, 2013)ํ․

En la segunda parte del texto, se reseñan tres programas de carácter nacional cuyos contenidos se relacionan con la promoción de una cultura de la legalidad y de la construcción de ambientes escolares seguros y libres de violencia. Se presentan para evidenciar, por un lado, que en los enfoques analíticos y en las intervenciones educativas el fenómeno de la violencia destaca como orientación temática $\mathrm{y}$, por el otro, que la mayor parte de las acciones está dirigida a la prevención y a la reducción de la violencia entre estudiantes; por consiguiente, se dejan al margen otros actores claves en los procesos formativos. También quedan relegadas del análisis las condiciones institucionales que limitan la transformación de prácticas de convivencia y de resolución de conflictos en las escuelas.

En tercer lugar, se toma postura respecto a la vertiente analítica que se asume en este artículo para reflexionar acerca de la problemática expuesta en los apartados anteriores. Esta se define desde la convivencia escolar con fundamento en los derechos humanos.

\section{Distintas miradas analíticas}

En México coexisten tres vertientes o vías para entender y explicar lo que acontece en las escuelas de educación básica sobre las interacciones humanas, los vínculos sociales y afectivos que se construyen; a saber: convivencia, disciplina y violencia (ver figura 1 ).

Aunque esas vertientes guardan relación, como puede observarse en el esquema, el conjunto de hallazgos y de aportaciones difiere, debido a la diversidad disciplinaria desde donde se realizan los acercamientos analíticos, las perspectivas y los métodos de investigación, así como los componentes que se priorizan en estos (actores, procesos y condiciones institucionales, por ejemplo). 


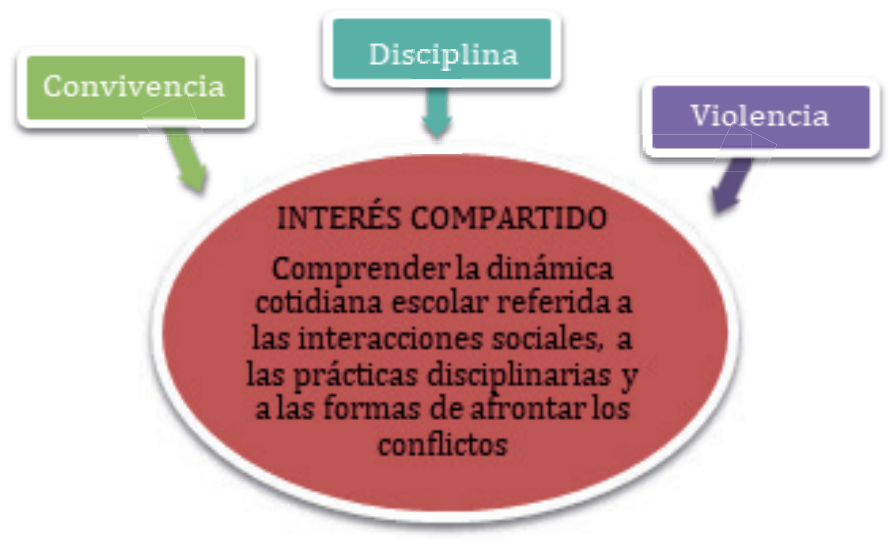

Figura 1. Tres vertientes temáticas. Fuente: elaboración propia con base en Furlán y Spitzer (2013).

La primera vía de análisis convivencia escolar se considera tema emergente porque es apenas, en la primera década del siglo XXI, cuando se ha identificado en el ámbito de las investigaciones e intervenciones educativas.

En el estado del conocimiento, de 1992 a 2002, su coordinador (Furlán, 2003) señalaba que, a pesar de su escasez en las publicaciones revisadas, la siguiente se incluyó como parte de la configuración del campo: Procesos y prácticas de disciplina y convivencia en la escuela. Los problemas de la indisciplina, incivilidades y violencia, por su trascendencia en España y Argentina. Textualmente señalaba:

en México casi nadie ha investigado el tema de la convivencia, porque la palabra no ha adquirido fuerza de uso en el ámbito escolar; sin embargo, si lo agregamos al campo, es porque viene aparejado con el tema de violencia y disciplina, por lo que en algún momento es probable que alguien centre su estudio en él (Furlán, 2003, p. 250).

Sin embargo, como lo anticipó Furlán (2003), actualmente la convivencia escolar es objeto de indagación y motivo de las intervenciones pedagógicas. Es complicado definir el término "convivencia escolar". De entrada, se observa la inexistencia de un concepto unívoco que facilite la delimitación de sus elementos. Su expresión remite a sucesos originados durante el encuentro con otros y a la coexistencia de los miembros de una comunidad escolar.

"Convivencia", también, seasocia con prácticas de interacción en instituciones educativas, con los criterios de organización escolar y las normas de convivencia, con el ejercicio del poder $y$ con la autoridad pedagógica, así como con los procesos de negociación y las formas de afrontar los conflictos interpersonales.

En el último estado del conocimiento, que corresponde al periodo 2002-2011, se observa un esfuerzo por acotar sus componentes y lograr precisión conceptual; para ello, se toma de referencia los documentos consultados (artículos de revistas, capítulos de libros, informes de investigación, memorias de congresos de investigación y jornadas educativas, tesis de 
posgrado, entre otros). De esta revisión, se obtienen dos modos de abordar el tema: 1) Convivencia como prevención de la violencia y 2) Convivencia como parte de la calidad de la educación. Asimismo, se anuncian los enfoques (inclusiva, pacífica y democrática) y las disciplinas presentes en su abordaje (Fierro, Lizardi, Tapia y Juárez, 2013).

Simultáneamente, otros términos próximos a convivencia se ubican en la literatura nacional: ambiente escolar y clima escolar. El primero se entiende como

el ámbito de aprendizaje, formación y convivencia ubicado en el espacio material y organizativo de la escuela. Está constituido por las distintas maneras de interacción entre docentes, directivos, alumnos, familias y personal de la escuela. Se conforma por los valores, las normas, las formas de convivencia y de trabajo que estos practican cotidianamente, así como en la forma en que se resuelven los conflictos, se comparten los espacios comunes, y en el trato que se da a niñas, niños y a los adultos de la escuela. (Secretaría de Educación Pública, 2008, p. 16).

¿Dónde se alude a ambiente escolar? En el Programa Integral de Formación Cívica Ética para la Educación Primaria que fue publicado por la Secretaría de Educación Pública en el 2008. Ahí se define como uno de los cuatro ámbitos de formación para informar que la intervención pedagógica no debe limitarse a un espacio curricular (la asignatura) ni a un enfoque transversal, debido a que los aprendizajes relacionados con aprender a ser y a vivir juntos se adquieren mediante la experimentación y la vivencia. De esta manera:

la organización de la escuela como institución, debe convertirse en fuente de aprendizaje de valores. Esto significa que la escuela como institución y el aula como microsociedad deben vivir relaciones interpersonales y formas de tomar decisiones acordes con los valores que los alumnos deben descubrir. (Schmelkes, 1999, p. 165).

El clima escolar se localiza en estudios sobre el derecho a la educación y el logro académico. Se define como un conjunto de factores presentes en las instituciones educativas que influyen en el aprendizaje de la comunidad estudiantil y, por tanto, en los resultados educativos (Blanco, 2009; Juárez y Fierro, 2013). Entre los aspectos destacables, están los estilos y las prácticas docentes que favorecen la construcción del conocimiento en el aula, los atributos o los rasgos docentes más valiosos para el alumnado, las condiciones institucionales que promueven la libertad y el espíritu de colaboración, entre otros.

La segunda vía analítica, disciplina escolar, aparece en asuntos relacionados con el ejercicio de la autoridad y el poder en las escuelas mediante normatividad escolar, la aplicación de marcos de convivencia y prácticas disciplinarias. Se le asocia, también, con el establecimiento de ciertos criterios y límites para conservar un orden mínimo en el sistema escolarizado, así como un componente estratégico a fin de resolver divergencias, disputas y conflictos escolares.

La mayoría de las veces disciplina escolar se encuentra articulada con convivencia escolar (Landeros y Chávez, 2015) y, en otras más, con ejercicios analíticos sobre violencia escolar. En este sentido, "disciplina escolar" funciona como una bisagra por su función intermedia entre convivencia y violencia. Si bien las investigaciones con énfasis en disciplina escolar han permanecido por más de dos décadas, su desarrollo es escaso e irregular. Los responsables del último estado del conocimiento se refieren a este punto en los siguientes términos:

se trata de un tema subvaluado, no sólo en México sino en otros contextos, pese a que la primera e inevitable tarea que todo docente debe ser capaz de realizar con éxito en la 
escuela, consiste en lograr que el grupo esté disponible para iniciar la clase. (Pereda, Plá y Osorio, 2013, p. 134).

La tercera vertiente analítica, violencia escolar, podría calificarse como la más consolidada por la producción constante durante más de diez años, aunque su estudio inició aproximadamente en los años 1990. No obstante, los responsables de revisar la generación de conocimiento desde esta perspectiva temática señalan que, dada la magnitud del problema, los avances logrados son magros (Gómez y Zurita, 2013, p. 184). Entre los temas objeto de análisis y de compresión, se encuentran: el papel de las nuevas tecnologías como espacios de socialización de niños, adolescentes y jóvenes, el bullying, el acoso escolar entre estudiantes.

La mirada se centra en actitudes y en comportamientos violentos entre estudiantes, y se ignoran otros miembros de la comunidad educativa (sobre todo adultos) que pueden ser protagonistas de actos de violencia, o bien víctimas de ella.

En este orden de ideas, se enfatiza la violencia directa y se aborda escasamente la cultural asimilada por las personas a lo largo de sus vidas y con independencia de la condición social y económica de origen. Entre este tipo de prácticas arraigadas en la convivencia, se hallan: el racismo, el sexismo, la intolerancia religiosa, la política y el menosprecio de la diversidad sexual. Tampoco se coloca en el centro de la discusión aquellas prácticas institucionales que generan injusticias y afectan la dignidad humana (violencia estructural).

A este debate educativo, en los últimos años, se han agregado otros problemas sociales derivados de la inseguridad pública, la delincuencia y la corrupción. De esta manera, el tema de la seguridad escolar se combina con elementos de violencia directa y estructural.
De acuerdo con Furlán y Spitzer (2013) durante el periodo 2002-2011, los problemas de violencia ocuparon más la atención en la comunidad investigadora. Estos mismos autores afirman que existe una correlación entre las vertientes (convivencia, disciplina y violencia), los intereses de quien investiga y sus áreas académicas de pertenencia. Al respecto, señalan:

Si la entrada es pedagógica, la tendencia es a estudiar el problema centrándose en la disciplina; si es psicológica, la tendencia es a estudiar las manifestaciones violentas y los "factores de riesgo"; o si es socio-política, a estudiar la convivencia democrática y sus potencialidades para la formación ciudadana.

Desde hace algo más de una década, la preocupación pedagógica también contempla el concepto convivencia [...]. La convivencia es considerada por muchos uno de los "pilares" de la educación. Se trata de aprender nuevas formas de estar juntos, a partir del respeto y la tolerancia hacia los otros. (Furlán y Spitzer, 2013, p. 23).

A pesar de observarse ciertas imprecisiones en esta clasificación -a causa de una vertiente temática, por ejemplo: la violencia, puede abordarse desde un plano pedagógico, psicológico o sociopolítico (retomando la clasificación que los autores señalan)-, el planteamiento es útil para mostrar los énfasis encontrados en los diversos escritos, así como la evidente diversidad conceptual o anarquía semántica, según se quiera juzgar, que se presenta en la producción documentada.

Por su parte, cabe advertir que, en México desde la última década del siglo XX (1990-2000), el currículo oficial de la educación primaria y secundaria incluye contenidos que abonan a un ámbito de aprendizajes vinculados con ser y vivir juntos (Delors, 1997)2.

Aprender a vivir juntos conlleva establecer relaciones con los demás desde la no violencia. Si 
se piensa en las instituciones escolares, implica promover una convivencia pacífica y respetuosa de las diferencias para desarrollar el sentido de la cooperación, la solidaridad y la comprensión; que favorezca el descubrimiento del otro, la diversidad sociocultural y la interdependencia para identificar y contribuir al logro de objetivos comunes (Delors, 1997).

Aprender a ser se refiere al conocimiento y al cuidado de sí como punto de partida para que las personas estén en disposición de conocer y de valorar a los demás. Comprende el desarrollo y el bienestar integral del ser humano en toda su riqueza y complejidad, como "individuo, miembro de una familia y de una colectividad, ciudadano y productor, inventor de técnicas y creador de sueños" (Delors, 1997, p. 102). En términos de Yurén (2005), aprender a ser corresponde a

la dimensión existencial o dimensión de la construcción y cuidado del sí mismo (en griego: épiméleia) y aprender a vivir juntos a la dimensión sociomoral de la formación que se refiere a la relación con los otros en el mundo social. (p. 22).

Particularmente, estos contenidos se incorporan a través de la asignatura de formación cívica y ética; asimismo, mediante programas extracurriculares sobre educación para los derechos humanos, educación para la paz y resolución no violenta de conflictos, cultura de la legalidad y formación en valores para la democracia ${ }^{3}$. Las investigaciones acerca de estas orientaciones temáticas se han agrupado en otra área de conocimiento Educación y valores ${ }^{4}$, la cual se define como

Abarca las investigaciones y estudios que se ocupan de la dimensión ética de los sujetos, instituciones, prácticas, políticas y discursos filosóficos en el campo educativo, así como de las desplegadas para la formación valoral (en sus dimensiones ética, estética, y corporal, entre otras), el desarrollo moral, la formación para los derechos humanos y la ciudadanía, los valores profesionales y la ética profesional (Hirsch y Yurén, 2013, p. 33).

De modo que, es posible calificar a esta área o campo como una opción más con aportaciones complementarias a convivencia, disciplina y violencia escolar para comprender la problemática aquí planteada.

Como se anticipó en la introducción, de modo paralelo a esta diversidad temática en el terreno investigativo, existen varias acciones y programas derivados de la actual política educativa. En el siguiente apartado, se comentan tres programas nacionales cuyo destinatario principal es la población estudiantil para promover ambientes escolares seguros y libres de violencia.

\section{Programas y acciones estatales relacionados con convivencia, disciplina y violencia escolar}

Aproximadamente en la primera década del siglo XXI (2000-2010), en las escuelas públicas de educación básica, cobró fuerza un conjunto de acciones relacionadas con la promoción de ambientes escolares seguros y libres de violencia.

El énfasis de esas acciones se debió, entre otras cosas, a la creciente preocupación por el clima de inseguridad pública y la violencia relacionada con la delincuencia organizada y su impacto en los contextos escolares. Entre sus objetivos centrales, se encuentra la promoción del apego a la legalidad y el fortalecimiento del estado de derecho. Por su difusión en distintas escuelas del país y su impulso desde el propio sistema educativo nacional, se destacan los tres programas siguientes:

1) Programa Cultura de la Legalidad que surgió "en 1998 a raíz de un acuerdo binacional con el gobierno de Baja California y el estado de California de Estados Unidos de América, en la Ciudad de San Diego" (Secretaría de Educación y Cultura, 2010, p. 6). Su nombre original era 
Formación Ciudadana hacia una Cultura de la Legalidad y, en el ciclo escolar 2012-2013, se consolidó como asignatura estatal optativa para el nivel de educación secundaria en 29 entidades federativas (México Unido Contra la Delincuencia, 2012).

2) El Programa Nacional Escuela Segura con cobertura en escuelas de educación primaria y secundaria a partir del 2007 en diversas entidades federativas del país.

3) El Programa Nacional de Convivencia Escolar que tuvo su origen en el Proyecto a favor de la Convivencia Escolar (PACE) durante el ciclo escolar 2014-2015 (SEP, 2017) y que, actualmente, incluye al Programa Nacional de Escuela Segura.

Para el desarrollo de los tres programas, se cuenta con materiales didácticos dirigidos a las autoridades escolares (directivos, personal de apoyo y docentes), así como a las familias. A continuación, se reseña cada uno de ellos siguiendo el orden de enunciación establecido anteriormente.

Cultura de la legalidad. Es el nombre del espacio curricular (asignatura optativa) que se imparte a adolescentes en el nivel de educación secundaria. Se introdujo a las escuelas secundarias del norte del país con el apoyo y asesoría del Centro Nacional de Información Estratégica de Estados Unidos (NSIC-USA/ National Strategy Information Center, 2003) y la Oficina de Educación del Condado de San Diego (USA). El estado pionero fue Baja California.

En un documento que circula en la red informática de internet se explica lo siguiente:

En México, el programa surgió como una respuesta de las autoridades educativas del estado de Baja California ante el paulatino deterioro social, producto de fenómenos como la corrupción, la delincuencia y el crimen organizado en la zona fronteriza con los Estados Unidos. Atendiendo el compromiso social del ámbito educativo, y en coordinación con la Oficina de Educación del condado de San Diego (USA), inició un proyecto escolar con el fin de generar en los alumnos mecanismos de reflexión y análisis en torno al perjuicio de los actos ilícitos y en la necesidad de construir y consolidar el estado de derecho y la cultura de la legalidad (Subsecretaría de Educación Pública, s. f., p.1).

Entre los recursos didácticos clásicos del programa, se hallan: el libro de texto para el estudiantado, la antología para los docentes, la obra El señor de las Moscas de William Golding y la película Buenos muchachos (Goodfellas de Martin Scorsese). El material en sus orígenes se centraba en disuadir a la juventud de incorporarse en la delincuencia organizada con técnicas de carácter autoritario y punitivo que no admitían ningún tipo de incumplimiento de las normas escolares ni objeción a la autoridad. En dicho material, se retomaban los planteamientos del enfoque "tolerancia cero" desarrollado en la ciudad de Nueva York para atender asuntos de seguridad ciudadana. Tal enfoque se basaba en sancionar con severidad toda infracción normativa independientemente de la gravedad de la falta cometida. Por fortuna, fue modificándose con las aportaciones y la mirada crítica de los propios docentes participantes en el programa, así como con las de quienes eran responsables del currículo en la Secretaría de Educación Pública entre los años 2000 y 2006.

La tabla 1 muestra algunos ajustes de contenido que se hicieron, de manera gradual, a lo largo de 12 años aproximadamente. Estos se incorporaron al someter el programa a revisión curricular en la Secretaría de Educación Pública, con el fin de hacerlo congruente con la legislación educativa nacional. Asimismo, el programa se vio influenciado por el enfoque "educativo de formación cívica y ética" (1999), el cual diluyó su énfasis punitivo, destacó el sentido educativo parala construcción de ciudadaníay promoviólas 
Tabla 1

Comparación de temas del Programa Cultura de la Legalidad

En el 2000 el programa estaba organizado en cuatro bloques temáticos ${ }^{5}$, de manera similar al del 2006

El único cambio visible es el ubicado en el bloque 1

\section{Cultura de la legalidad}

Su objetivo es que el estudiantado construya y reflexione sobre el concepto de cultura de la legalidad, sobre las características que nos hacen humanos e individuos, así como sobre el sentido e importancia de las normas y los valores en la convivencia social.

\section{El estado de derecho y la cultura de la legalidad}

El énfasis de esta sección es sobre el estado de derecho. Los jóvenes aprenderán en qué consiste, cómo reconocerlo y por qué es importante protegerlo. Estarán en posibilidades de darse cuenta de que frente a normas, a leyes o a una impartición de justicia incorrectos, ellos mismos pueden propiciar un cambio. El enfoque principal será cómo y por qué es necesario mantener una cultura de legalidad.

III. El crimen y la corrupción: enemigos de la cultura de la legalidad

Se ocupa de los efectos perjudiciales que acarrea el crimen organizado a los individuos involucrados, a sus familias, a sus amigos y a la sociedad entera. El estudiantado aprenderá qué es el crimen organizado, cómo funciona, cómo atrae a nuevos miembros y por qué representa una amenaza a la sociedad en general. Además, la comunidad estudiantil empezará a reflexionar sobre los medios legales e institucionales para combatir la delincuencia.
La publicación 2006 del mismo programa con aprobación de la Secretaría de Educación Pública consideraba cuatro bloques con algunas variantes muy tenues, en términos de contenidos ${ }^{6}$

\section{Individuo y sociedad}

El propósito de este capítulo es que el alumnado, mediante la reflexión y el conocimiento de sí mismo, se identique como un ser social y sea capaz de contribuir a la convivencia de la sociedad viviendo una cultura de la legalidad.

\section{El estado de derecho y la cultura de la legalidad}

El énfasis de esta sección es sobre el estado del derecho. Los jóvenes conocerán las características del estado de derecho y de la cultura de la legalidad y reconocerán las ventajas de ponerlo en práctica en su vida cotidiana.

III. Delincuencia y corrupción: elementos que atentan contra la cultura de la legalidad

Se ocupa de los efectos perjudiciales que acarrea el crimen organizado a los individuos involucrados, a sus familias, a sus amigos y a la sociedad entera. El estudiantado aprenderá qué es el crimen organizado, cómo funciona, cómo atrae a nuevos miembros y por qué representa una amenaza a la sociedad en general. Además, la comunidad estudiantil empezará a reflexionar sobre los medios legales e institucionales para combatir la delincuencia.

\section{Derechos humanos, educación para la} paz y cultura de la legalidad

Que el alumnado se familiarice con los derechos humanos, que los asuma como propios y los acate para garantizar el respeto de los derechos de otros.

Continúa... 
IV. Promoviendo una cultura de legalidad

Esta última sección le permite al estudiantado ver su papel en la promoción y el apoyo del estado de derecho. Se estimula el rechazo al crimen organizado y a sus actividades; además, se intenta crear conciencia sobre su responsabilidad para actuar en este rubro. Se le estimula a pensar en su propio futuro y en las consecuencias de las decisiones que toman. Por último, se les enseña técnicas de resolución de problemas, para ayudarlos a vencer los obstáculos que enfrenten en la búsqueda de sus metas.
IV Promoviendo una cultura de legalidad y un proyecto de vida

4. Estado de derecho, cultura de la legalidad y sentido de justicia

Esta última sección le permite al estudiantado ver su papel en la promoción y el apoyo del estado de derecho. Se estimula el rechazo al crimen organizado y a sus actividades; además, se intenta crear conciencia sobre su responsabilidad para actuar en este rubro. Se le estimula a pensar en su propio futuro y en las consecuencias de las decisiones que toman. Por último, se le enseñan técnicas de resolución de problemas, para ayudarlos a vencer los obstáculos que enfrentan en la búsqueda de sus metas.
Que el alumnado adquiera conocimientos y desarrolle las competencias ciudadanas que le permitirá identificar y rechazar prácticas que amenazan los derechos fundamentales, la cultura de la legalidad y el estado de derecho.

5. Participación, ejercicio de ciudadanía y construcción de una cultura de la legalidad

Que alumnado cuente con los elementos necesarios para participar en decisiones que afectan la vida escolar y comunitaria, y participe en un verdadero ejercicio de ciudadanía, construcción de la cultura de la legalidad y fortalecimiento del estado de derecho.

Fuente. Secretaría de Educación Pública, s. f.; Secretaría de Educación Pública SEP, 2006; México Unido Contra la Delincuencia, s. f.

capacidades de autorregulación y empatía para producir un aprendizaje sociomoral centrado en el sentido de justicia y apego a la legalidad.

Como puede observarse, los aspectos relacionados con el fortalecimiento de un estado de derecho democrático reemplazaron el énfasis en la prevención de delitos y el crimen organizado; asimismo, la cultura de la legalidad se vinculó con la construcción de ciudadanía.

Programa Nacional de Escuela Segura (PNES). Se puso en marcha durante el ciclo escolar 2007-2008 en nueve entidades federativas para responder a problemas de seguridad pública: violencia, delincuencia, narcotráficoy adicciones. Desdesus inicios es de cobertura nacional y se incorporan a él, de forma voluntaria, las escuelas. Para efectos de la formalización, se establece un convenio de trabajo y coordinación con la Secretaría de Educación Pública (SEP). Sin embargo, en las Reglas de Operación del Programa de Escuela Segura, se indica una atención prioritaria para escuelas ubicadas en zonas de mayor incidencia delictiva. Esto se puede observar en algunos apartados de las Reglas de Operación del Programa de Escuela Segura en distintos años, 
aunque con matices distintos. Sirva de ejemplo lo publicado en el Diario Oficial de la Federación 2008 y, posteriormente, en el 2013. El acuerdo 476 por el cual se emiten las Reglas de Operación del Programa de Escuela Segura, 2008, señala en el punto 3.3.2 Procedimiento de selección:

La incorporación de las escuelas al PES es voluntaria, inicia con la inducción que lleva a cabo la CEPES. Prioritariamente se promueve el PES en las escuelas ubicadas en los 150 municipios de mayor incidencia delictiva, aunque la invitación se extiende al resto de los centros escolares de cada una de las entidades federativas (SEGOB, 2008).

Por su parte, el acuerdo 663 por el que se emiten las Reglas de Operación del Programa de
Escuela Segura, 2013, precisa en el apartado 4.2 Población Objetivo lo siguiente:

Escuelas públicas de educación básica en todos sus niveles y modalidades interesadas en llevar a cabo una agenda de convivencia y seguridad escolar que permita reducir la violencia y contribuya al desarrollo de habilidades para evitar el consumo de sustancias adictivas. La atención a estas escuelas se focalizará prioritariamente en los municipios y delegaciones políticas en el caso de Distrito Federal, de mayor riesgo de los 31 estados y el Distrito Federal (SEGOB, 2013).

Al hacer referencia a su desarrollo en la composición y operación del programa, la Secretaría de Educación Pública presenta su enfoque así (ver figura 2):

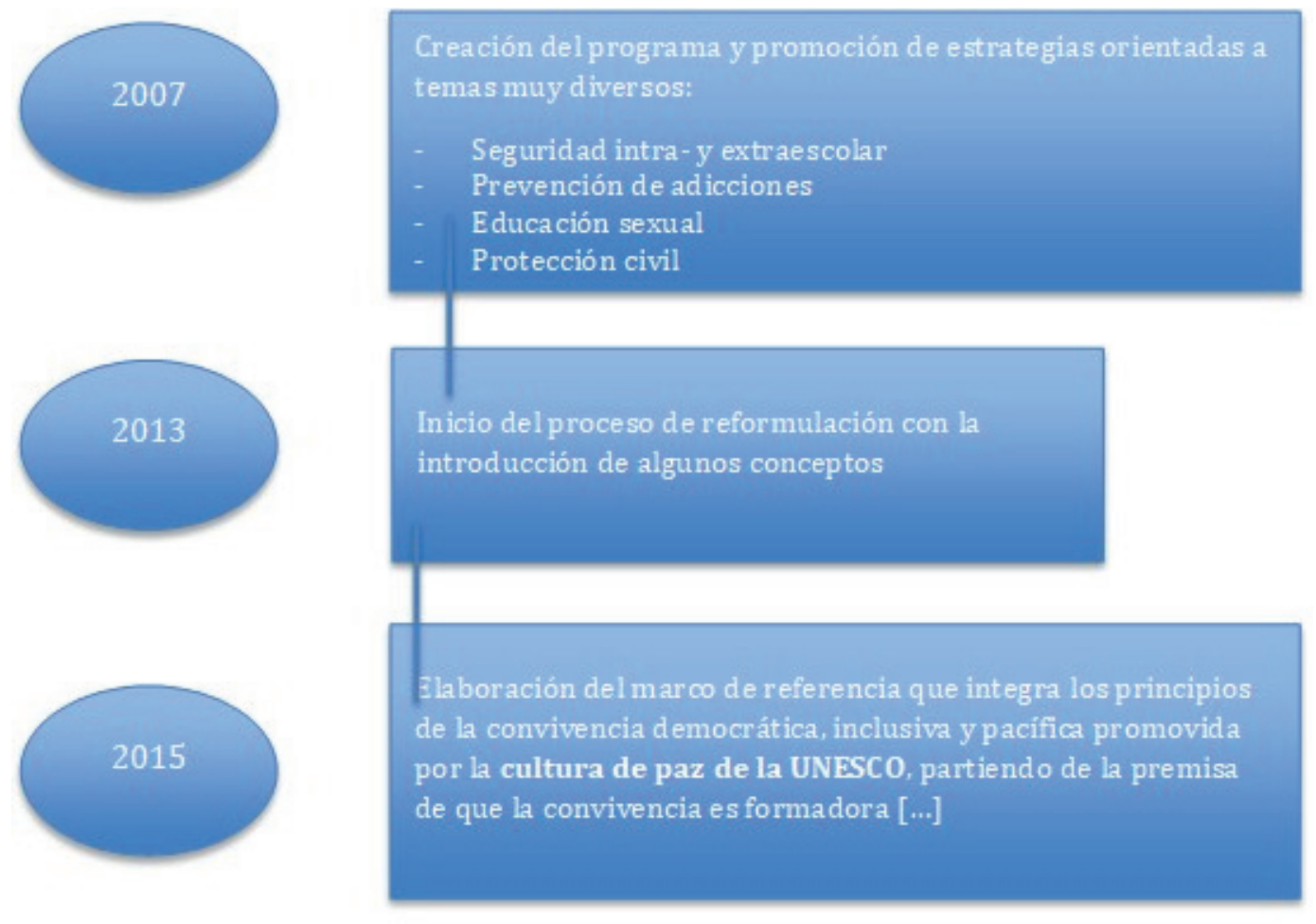

Figura 2. Enfoque del PES. Evolución de la operación del PES. Fuente. Secretaría de Educación Pública, 2018 
Así sucede la transición de un abordaje de asuntos mayormente vinculados con la prevención del delito y de adicciones hacia el fortalecimiento de una cultura de la prevención del riesgo, la construcción de ambientes protectores y el desarrollo de competencias ciudadanas mediante el currículo formal (asignaturas y transversalidad), la gestión escolar y la participación social.

Programa Nacional de Convivencia Escolar (PNCE). Actualmente es el prioritario y el Programa de Escuela Segura se ha incluido como parte de sus líneas de intervención. El PNCE se ha definido como "un Proyecto educativo, de carácter preventivo y formativo que consiste en la elaboración de materiales didácticos para apoyar el trabajo académico que maestros y alumnos realizan en el salón de clase. Forma parte de una extensión curricular de la asignatura de Formación Cívica y Ética" (SEP, s. f.), y su propósito es "contribuir al fortalecimiento de una convivencia inclusiva, democrática y pacífica" (SEP, s. f.).

De acuerdo con información de la propia Secretaría de Educación Pública (2017), el Programa Nacional de Convivencia Escolar se encuentra en 21000 escuelas de primero a sexto de primaria. Para el ciclo escolar 2018-2019, la meta proyectada incluye la cobertura de "más de 80000 escuelas de todo el país de educación básica: preescolar, primaria y secundaria" (SEP, s.f.). Entre los criterios para su selección, son similares a los previstos para el PNES de años anteriores, al menos los revisados para el ejercicio fiscal 2016:

Escuelas Públicas de Educación Primaria, que preferentemente se ubiquen en las demarcaciones y polígonos del [Programa Nacional para la Prevención Social de la Violencia y la Delincuencia] PNPSVyD. En caso de existir disponibilidad presupuestaria, el PNCE se podrá extender a escuelas públicas de educación primaria ubicadas en otras demarcaciones, que hayan manifestado, mediante una Carta Compromiso Escolar dirigida a la AEL, su interés y compromiso por participar en el Programa. (SEGOB, 2015, p. 70).

El primer programa comentado es una asignatura optativa bajo la responsabilidad de un docente y los dos siguientes son opciones extracurriculares que implican la incorporación del colectivo docente en el desarrollo de acciones específicas; sin embargo, a pesar de estas diferencias, parecen compartir motivaciones y preocupaciones, así como estrategias y enfoques educativos. A ellos se hará referencia en el siguiente apartado.

Enfoques presentes en programa y acciones educativas

Los programas y acciones educativas, de acuerdo con sus rasgos principales pueden clasificarse en dos tipos: Enfoque remedial y Enfoque preventivo y formativo (PREAL, 2003). Esta clasificación se retoma porque se vincula con lo recién expuesto acerca de los dos modos de abordar el tema de la convivencia escolar (Fierro, Lizardi, Tapia y Juárez, 2013): 1) Convivencia como prevención de la violencia, se aproxima al enfoque remedial; y 2) Convivencia como parte de la calidad de la educación, con el segundo, esto es, el enfoque preventivo y formativo.

Enfoque remedial. Se centra en evitar ciertas manifestaciones de violencia directa y prácticas específicas como violencia entre pares, bullying, indisciplina, acoso sexual y violencia en redes sociales(ciberbullying). Laintervenciónseorienta hacia un grupo de niños, niñas y adolescentes que exhiben conductas disruptivas y quienes sobresalen en situaciones de indisciplina y agresión entre compañeros. En este modo de afrontar los desafíos de la convivencia en la escuela, frecuentemente, se atribuye la causa del problema al entorno familiar y social donde se desarrollan los menores de edad. Siguiendo 
el planteamiento inicial de PREAL (2003), este enfoque se relaciona con las estrategias de carácter restringido en Estados Unidos y Brasil a principios del siglo XXI.

Enfoque preventivo y formativo. Si bien en el anterior destacan condiciones negativas y adversas que deben controlarse, este se fundamenta en una explicación más compleja de la realidad; considera factores externos (individuales, familiares, sociales, económicos y culturales) e internos al ámbito escolar (PREAL, 2003) para promover una convivencia pacífica, solidaria e incluyente.

Aunque en sus orígenes los tres programas descritos correspondían con el primer enfoque, de manera gradual, se está logrando transitar a uno menos reactivo y punitivo. Durante los últimos años, se han hecho esfuerzos de distinto tipo, tanto en los colectivos docentes como en los equipos técnico-pedagógicos de la Secretaría de Educación por aproximarse al enfoque preventivo y formativo.

¿En qué regiones o zonas del país, principalmente, se están desarrollando estos programas?, ¿cómo se seleccionan las escuelas?, ¿cuáles son los destinatarios prioritarios?, ¿por qué? Estas preguntas se formulan con el ánimo de llamar la atención respecto a un planteamiento destacado en dos escritos anteriores (Chávez, 2014; Latapí, 2003). En el primero, se reflexiona respecto al enfoque de riesgo vinculado a la población adolescente y juvenil de sectores urbano-marginales y populares, a quien "se asocia con la pertenencia a un grupo generador de problemas que tienen que ver con la violencia, la delincuencia, la drogadicción y el ejercicio irresponsable de la sexualidad" (Chávez, 2014, p. 5). Este enfoque ha estado presente en América Latina desde hace varias décadas y parece conservarse en la construcción de programas dirigidos a esta población.
En el segundo, se hace referencia a los criterios de selección de escuelas para desarrollar programas orientados a la formación de valores y se advierte que las de mayor intervención se encuentran en condiciones de vulnerabilidad (Latapí, 2003, p. 173). Esta práctica aparece de modo recurrente y sobre ella se ha llamado la atención en diversos momentos sin observarse ningún cambio. Permanece como una línea de interpretación y de justificación de acciones educativas diversas.

Una vez mostradas las tres vertientes principales, en México, desde donde es posible introducirse a la investigación o al análisis de programas y de acciones educativas, así como los programas vinculados con convivencia, disciplina y violencia escolar conviene destacar la vía analítica adoptada para la generación y aplicación de conocimiento de quien ha elaborado el presente escrito. También, se hace con el propósito de abonar a la discusión.

Así pues, se reitera que no se trata de descartar la diversidad de opciones analíticas; sí, en cambio, de precisar sus contribuciones, delimitar sus propósitos y apoyar en su proceso de consolidación como perspectivas analíticas complementarias. Esto podría favorecer una comprensión integral y multidimensional de los problemas de la convivencia escolar, siempre y cuando se logre un desarrollo equilibrado y sistemático. Sin embargo, como se ha dicho, esto no parece estar sucediendo. Al menos en el caso las investigaciones realizadas en la primera década del siglo XX (entre los años 2002 y 2011), se observa un mayor interés por analizar el fenómeno de la violencia y, peor aún, solo entre estudiantes.

La convivencia escolar vista desde la perspectiva de los derechos humanos

De las tres vertientes presentadas en el primer apartado, para quien suscribe estas reflexiones, 
la convivencia escolar es la preferida para pensar en los procesos formativos y la construcción de ciudadanía en las escuelas de educación básica (preescolar, primaria y secundaria). Concomitantemente, se considera una vía para analizar y comprender las políticas educativas, así como la organización escolar y los procesos de formación, desde una perspectiva éticopolítica que reconoce a la educación como un derecho fundamental (Tomasevski, 2004) para el desarrollo integral de las personas y la formación de ciudadanía.

Cuando se asume una perspectiva con fundamento en los derechos humanos para el contexto escolar, se reconoce la dignidad humana como un elemento compartido por los integrantes de la comunidad educativa (estudiantes, docentes, personal directivo, asesores o personal con funciones de orientación y tutoría, familias). En este sentido, tanto las personas adultas como la población infantil y juvenil son sujetos de derechos, lo cual implica reconocerles, sin distinción, la capacidad de goce de bienes y satisfactores fundamentales para su bienestar personal y colectivo; sean ciudadanos o no, capaces de obrar o no. La noción de dignidad humana es la fuente de la que derivan los derechos básicos, primarios fundamentales, según se les quiera denominar.

De acuerdo con Habermas (2010), "la idea de la dignidad humana sirve como un 'portal' a través del cual la sustancia igualitaria y universalista de la moral se traslada al derecho" (p. 3) y sostiene la tesis de que "siempre ha existido - aunque inicialmente solo de modo implícito - un vínculo conceptual interno entre los derechos humanos y la dignidad humana" (Habermas, 2010, p. 6).

Por su parte, Ferrajoli (2005) define como derechos fundamentales a "aquellos derechos subjetivos que las normas de un determinado ordenamiento jurídico atribuyen universalmente a todos en tanto personas, ciudadanos y/o personas capaces de obrar" (p. 292) y advierte que son tres los rasgos o características que los distinguen de los demás: su forma universal; su estatuto de reglas generales y abstractas; su carácter indisponible e inalienable.

¿Qué significa incorporar la perspectiva de los derechos humanos en el contexto escolar? En síntesis, requiere tomar en cuenta al menos tres componentes básicos: la defensa y la promoción de la dignidad humana, el ejercicio de las libertades fundamentales y la oposición al ejercicio unilateral o autoritario del poder. Siguiendo los planteamientos de la pedagogía radical, implica el reto de el reto de transformar prácticas de dominación y de autoritarismo que se reproducen mediante la escolarización en acciones que generen procesos de resistencia y de emancipación (Giroux, 1997).

De esta manera, se colocan al centro de la discusión los siguientes puntos:

1) el papel de la autoridad docente en la resolución de conflictos y las formas de ejercer su poder.

2) La función de la autoridad pedagógica para el desarrollo de la autonomía moral (la apropiación o la construcción de la norma).

3) Las contribuciones del docente a partir del vínculo pedagógico y del desarrollo de sus clases.

Una opción educativa con fundamento en los derechos humanos, de manera natural, rechaza el ejercicio de un poder autoritario porque, bajo esta perspectiva, se concibe a la escuela no como un espacio para la reproducción, sino para la transformación social, y tal pretensión requiere formar sujetos autónomos, libres y responsables. Esto es imposible de impulsar desde cualquier estilo de intervención educativa y tipo de organización escolar. 
Al hablar de la convivencia escolar ${ }^{8}$, es necesario explicitar dos elementos que la componen: por un lado, el logro, en las escuelas, de ciertas condiciones disciplinarias, organizacionales y de infraestructura para que suceda el acto educativo; y por el otro, el reconocimiento de la convivencia escolar como un aprendizaje en sí mismo, porque incluye el proceso de adquisición de un conjunto de conocimientos vinculados directamente con dos de los cuatro pilares de la educación (Delors, 1997): aprender a ser y a vivir juntos.

Las vías de aprendizaje para la convivencia son diversas. Una de ellas se articula al currículo prescrito (en las asignaturas y el manejo transversal de contenidos); y la otra, a las interacciones espontáneas que ocurren en los diversos espacios de la institución escolar, por ello, es imposible limitarla al espacio del aula. Por esta razón, los modos de gestionar las divergencias, los conflictos interpersonales y el análisis de los ambientes de aprendizaje instalados como parte de la cultura escolar se colocan en el centro del debate.

La convivencia implica el reconocimiento de tensiones en la vida diaria por la diversidad de intereses y de necesidades que confluyen en un espacio compartido. Asimismo, requiere establecer criterios normativos que protejan las libertades fundamentales y regulen la actuación individual para asegurar el respeto a los derechos de terceros, sean autoridades escolares; personal con funciones de docencia, orientación o tutoría; familias, niños, niñas o adolescentes.

En el ámbito escolar, aprender a convivir significa reconocer que, en el centro de la convivencia, se ubican los conflictos, no la violencia; y que las formas de afrontarlos pueden ser diversas: por vías pacíficas (cuando se antepone la negociación, la mediación y conciliación) o vías violentas (cuando se ignora o evadeelconflictoosedejaallibrejuegodefuerzas).
Una de las finalidades de la escuela, con frecuencia ignorada en la práctica, es el desarrollo de capacidades de sus integrantes, a saber: las sociales y afectivas para ser y estar con otros. De aquí la relevancia de analizar las normas escolares y las prácticas específicas de socialización, el ejercicio de la autoridad pedagógica, los mecanismos de participación y la toma de decisiones en las escuelas, las estrategias y los modos de dirimir los conflictos entre diversos actores. Estos aspectos son básicos para la comprensión integral de la convivencia.

El conflicto es inherente a las relaciones humanas, colocarlo en el centro del análisis permite complejizar la mirada sobre la diversidad de asuntos que se entretejen como parte de la cultura escolar.

Por su parte, las escuelas de educación básica necesitan propiciar un ambiente de aprendizaje para promover la participación del alumnado en la toma de decisiones, asumir el diálogo como un procedimiento de alto valor formativo en la construcción de consensos, aprender a respetar el disenso y reconocer el pluralismo como un valor clave para la vida ciudadana. Para todo lo anterior, se requieren autoridades escolares capaces de orientar su actuación, tanto en la relación pedagógica como en la resolución de conflictos, con base en criterios de justicia, libertad e igualdad en derechos.

\section{Conclusiones}

El propósito de estas reflexiones consistió en exponer las distintas rutas analíticas que han surgido en más de dos décadas en México, para comprender la dinámica cotidiana escolar referida a las interacciones sociales, prácticas disciplinarias y formas de afrontar los conflictos, ya sea por vías pacíficas o violentas.

En la mayoría de las investigaciones educativas mexicanas, en la última década, se le ha dado prioridad a la comprensión de la 
violencia escolar entre pares (bullying y acoso escolar) y se han marginado otros aspectos y actores clave responsables del acto educativo. Independientemente de la vertiente analítica que se escoja: convivencia, disciplina y violencia, la comprensión de los desafíos de la convivencia y las prácticas de interacción en las escuelas amerita un abordaje multidisciplinario. Este, de alguna manera, podría contribuir a una mejor intervención educativa.

En el plano de las intervenciones educativas, se encuentra el desarrollo de programas y de estrategias diversas, con una perspectiva restringida, en parte, como consecuencia de la escasez de estudios. Esta situación reduce las oportunidades de atención efectiva de los problemas por afrontar o superar.

Finalmente, el reconocimiento de las tres vertientesanalíticasexhibequelasinvestigaciones nacionales con énfasis en convivencia escolar destacan el papel de las instituciones escolares en la construcción de ciudadanía; función que, en el ámbito de la educación obligatoria, se le asigna al estado mexicano desde la propia Constitución Política de los Estados Unidos Mexicanos. Por lo tanto, desde el enfoque de derechos humanos, se prioriza la construcción de ambientes escolares que brinden oportunidades, a niñas, a niños y a adolescentes, de vivir, experimentar un buen trato con base en principios de la igualdad en derechos, el respeto a la diversidad, la búsqueda de la equidad y la justicia y la convivencia solidaria; así como, el desarrollo de capacidades para resolver los conflictos interpersonales o grupales por vías no violentas.

\section{Referencias}

Chávez, C. (enero-junio, 2014). La estigmatización de la adolescencia como grupo poblacional en riesgo y sus repercusiones en la construcción de ciudadanía. Sinéctica, 42. Recuperado de http://www.sinectica.iteso.

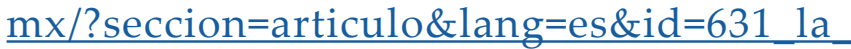
estigmatizacion de la adolescencia como grupo

Chávez, C. (2017). Premisas básicas para pensar la convivencia escolar desde una perspectiva democrática. En J. C. Carozzo y E. Arévalo, Una visión Iberoamericana de la convivencia escolar (pp. 67-86). Lima: Fondo Editorial de la Universidad Privada Antenor Orrego.

Delors, J. (coord.) (1997). La educación encierra un tesoro. Informe a la UNESCO de la Comisión Internacional sobre la Educación para el siglo XXI. Paris: UNESCO.

SEGOB (Secretaría de Gobernación) (31 de diciembre de 2008). Acuerdo número 476 por el que se emiten las Reglas de Operación del Programa de Escuela Segura. Diario Oficial de la Federación. México, DOF. Recuperado de http://dof.gob.mx/nota detalle.php?co$\underline{\text { digo }}=5076675 \&$ fecha $=31 / 12 / 2008$

SEGOB (25 de febrero de 2013). Acuerdo número 663 por el que se emiten las Reglas de Operación del Programa de Escuela Segura. Diario Oficial de la Federación. México. Recuperado de http://www.dof.gob. $\underline{\mathrm{mx} / \text { nota detalle.php? codigo }=5288861 \& \mathrm{fe}-}$ cha $=25 / 02 / 2013$

SEGOB (27 de diciembre de 2015). Acuerdo número 19/12/15 por el que se emiten las Reglas de Operación del Programa Nacional de Convivencia Escolar para el ejercicio fiscal 2016. Diario Oficial de la Federación. México. Recuperado de http://www.gob. $\mathrm{mx} / \mathrm{cms} /$ uploads/attachment/file/46116/ convivencia.pdf

Ferrajoli, L., L. Baccelli, M. Bobero, R. Guastini, M. Jori, A. Pintore, E. Vitale y D. Zolo (2005). Los fundamentos de los derechos fundamentales. En L. Ferrajoli Los fundamentos de los derechos fundamentales (pp. 287381). Madrid: Trotta. 
Fierro, C., A. Lizardi, G. Tapia y M. Juárez (2013). Convivencia Escolar. Un tema emergente de investigación educativa en México. En A. Furlán y T. Spitzer (coords.). Violencia en las escuelas. Problemas de Convivencia y Disciplina (pp. ). México: COMIE/ANUIES.

Furlán, A. (coord.) (2003). Procesos y prácticas de disciplina y convivencia en la escuela. Los problemas de la indisciplina, incivilidades y violencia. En J. M Piña, A. Furlán y L. Sanudo. Acciones, actores y prácticas educativas (pp. 245-398). México: COMIE/SEP/CESU.

Furlán, A. y T. Spitzer (coords.) (2013). Violencia en las escuelas. Problemas de Convivencia y Disciplina. Colección Estados del Conocimiento 2002-2011. México: COMIE/ ANUIES.

Gómez, A. y U. Zurita (2013). El estudio de la violencia escolar, orígenes y tendencias. En A. Furlán y T. Spitzer (coords.), Convivencia, disciplina y violencia en las escuelas 2002-2011 (pp. 183-222). México: ANUIES, COMIE.

Giroux, H. (1997). Teoría y resistencia en educación. México: Siglo Veintiuno editores.

Habermas, Jürgen (mayo 2010). El concepto de dignidad humana y la utopía realista de los derechos humanos. Diánoia LV (64), 3-25. Recuperado de http://www.scielo.org. mx/pdf/dianoia/v55n64/v55n64a1.pdf

Hirsch, A. y T. Yurén (2013). La investigación en México en el campo Educación y valores 20012011. Colección Estados del Conocimiento. México: COMIE/ANUIES.

Juárez, M. y C. Fierro (2013). Clima escolar. En A. Furlán y T. Spitzer (coords.), Violencia en las escuelas. Problemas de Convivencia y Disciplina (pp 107-112). Colección Estados del Conocimiento. México: COMIE/ ANUIES.

Landeros, L. y C. Chávez (2015). Convivencia y disciplina en la escuela. Análisis de reglamentos escolares de México. México: INEE. Recuperado de http://publicaciones.inee.edu.mx/ buscadorPub/P1/C/232/P1C232.pdf

Landeros, L. (2016). Formación cívica y ética: la configuración de un discurso (Tesis doctoral). Universidad Nacional Autónoma de México, México.

Latapí, P. (2003). (2003). El debate sobre los valores en la escuela mexicana. México: Fondo de Cultura Económica.

México Unido Contra la Delincuencia (s. f.). Asignatura Estatal Cultura de la legalidad Segunda Antología comentada. México: MUCD.

National Strategy Information Center (2003). Educación escolar por una cultura de la legalidad. Guía para el docente tercer grado de secundaria. México: Poder Ejecutivo de Baja California y Sistema Educativo.

Pereda, A., S. Plá y E. Osorio (2013). Disciplina e indisciplina en la escuela. Un estado del conocimiento. En A. Furlán y T. Spitzer. (coords.), Convivencia, disciplina y violencia en las escuelas 2002-2011 (pp. 133-181) Colección Estados del Conocimiento 2002-2011. México: ANUIES, COMIE.

PREAL (octubre, 2003). Estrategias para la prevención de la violencia y promoción de una cultura de paz en las escuelas, 1, 1. Santiago de Chile: Programa de Promoción de la Reforma Educativa en América Latina.

Secretaría de Educación Pública (s. f.). Programa Nacional de Convivencia Escolar. Recuperado de https://www.gob.mx/cms/uploads/ attachment/file/147561/Programa_Nacional de Convivencia Escolar.pdf).

Secretaría de Educación Pública (1999). Formación Cívica y Ética. Programas de estudio comentados. Educación secundaria. México: SEP.

Secretaría de Educación Pública (2006). Formación Ciudadana. Hacia una cultura de la legalidad. 
Situaciones sociales de impacto en la vida de los adolescentes. México: SEP.

Secretaría de Educación Pública (2008). Programa Integral de Formación Cívica Ética para la Educación Primaria. México: SEP.

Secretaría de Educación Pública (2018). Enfoque del PES. Evolución de la operación del PES. Recuperado de http://basica.sep.gob.mx/publications/pub/321/Enfoque+del+PES

Secretaría de Educación Pública (s. f.). Formación Ciudadana Hacia una Cultura de la Legalidad. Programa Prometedor. México: Subsecretaría de Educación Pública. Recuperado de www.oas. org/udse/cddemocracia/espanol/mexico2.doc

Schmelkes, S. (1999). Cinco premisas sobre la formación de valores. En G., Papadimitriou.
Programa de Educación en los Valores de la Paz y los Derechos Humanos. Antología (pp.157-168). México: ILCE-AMNU.

Tomasevski, K. (2004). Indicadores del derecho a la educación. Revista del Instituto Interamericano de Derechos Humanos, 40, 341-488. Recuperado de http://www.juridicas.unam.mx/publica/ librev/rev/iidh/cont/40/pr/pr18.pdf

Yurén, T. (2005). Ethos y autoformación en los dispositivos de formación de docentes. En T. Yurén, C. Navia y C. Saenger (coords.), Ethos y autoformación del docente. Análisis de los dispositivos de formación de profesores (pp. 19-45). México: Ediciones Pomares.

Recibido: 31 de mayo de 2018 Aceptado: 24 de setiembre de 2018

\footnotetext{
${ }^{1}$ En México desde el año 1996, se ha editado una colección de libros cada 10 años, con el propósito de realizar un balance de las investigaciones educativas en el país. En la primera edición, se hizo un recuento de la producción de conocimiento correspondiente al período 1982-1992 y, como consecuencia de ese trabajo, se fundó El Consejo Mexicano de Investigación Educativa (COMIE), la Asociación Civil, establecida el 23 de septiembre de 1993, que reúne a más de 500 investigadores pertenecientes a 202 instituciones (públicas y privadas) en 30 estados de la República. Para más información, puede consultarse la página web, http://www.comie.org.mx/v5/sitio/historia/. ${ }^{2}$ Cabe recordar que, de acuerdo con el Informe a la UNESCO de la Comisión Internacional sobre la Educación para el siglo XXI coordinada por Jacques Delors, publicado bajo el título de La educación encierra un tesoro (1997), los cuatro pilares de la educación son aprender a conocer, aprender a hacer, aprender a vivir juntos y aprender a ser. Sin embargo, en el caso de la educación básica en México, con frecuencia, se observan dificultades para asegurar un tratamiento integral de los cuatro pilares, e incluso se llama la atención respecto a que los dos últimos son escasamente atendidos, a pesar de la existencia de programas oficiales que guardan relación con estos.

${ }^{3}$ Un recorrido exhaustivo acerca de los énfasis temáticos que se asocian a la construcción de ciudadanía se encuentran documentados en Latapí (2003) y Landeros (2016).

${ }^{4}$ Dicha área se compone de siete subáreas, una de ellas formación ciudadana y derechos humanos.

${ }^{5}$ Información recuperada textualmente de www.oas.org/udse/cddemocracia/espanol/mexico2.doc (consulta 4 abril, 2018).

${ }^{6}$ Información tomada textualmente del documento SEP (2006) Formación Ciudadana hacia una Cultura de la Legalidad. Presentación del Programa 2006. Dirección General de Desarrollo de la Gestión e Innovación Educativa Dirección de Innovación Educativa Coordinación Nacional de Formación Ciudadana.
} 


\section{Chávez Romo}

${ }^{7}$ Información extraída de México Unido Contra la Delincuencia (s. f.). Asignatura Estatal Cultura de la legalidad Segunda Antología comentada, p. V.

${ }^{8}$ La precisión conceptual sobre el significado de la convivencia escolar que aquí se expone es retomada de Chávez, C. (2017). "Convivencia escolar y estrategias docentes en una escuela secundaria" XIV Congreso Nacional de Investigación Educativa-Ponencia. 